\title{
Manejo del paciente en atención odontológica y bioseguridad del personal durante el brote de coronavirus SARS-CoV-2 (COVID-19).
}

\author{
Patient management in dental care and staff biosecurity during \\ the SARS-CoV-2 coronavirus outbreak (COVID-19).
}

\author{
Carlos Bermúdez-Jiménez,* César Gaitán-Fonseca,* Luis Aguilera-Galaviz*
}

\section{RESUMEN}

El surgimiento de infecciones originadas por el SARS-CoV-2 en el humano ha desencadenado una serie de conflictos de salud, económicos y sociales en el entorno mundial. El área odontológica debe poseer todo el conocimiento necesario acerca de esta pandemia debido a que, como profesionales de la salud y responsables de la condición bucal de la sociedad, es de vital importancia disminuir el riesgo que presentan las enfermedades crónicas no transmisibles, cáncer, obesidad y otras, así como de los adultos mayores para que no generen un problema de salud severo y que además puedan ser asociadas con la presencia del COVID-19. Es importante conocer cuáles son las pautas por considerar en la práctica odontológica para una atención odontológica oportuna y eficaz manteniendo la bioseguridad del personal de salud. Por lo tanto, se genera esta guía de atención odontológica basada en la evidencia científica publicada para el conocimiento y dominio del profesional de salud oral.

Palabras clave: COVID-19, coronavirus, odontología, manejo, bioseguridad, salud pública.

\section{INTRODUCCIÓN}

$\mathrm{E}$ I 8 de enero de 2020 fue anunciada a nivel mundial una nueva cepa de coronavirus como la causa patógena del COVID-19 por el centro chino para el Control y Prevención de Enfermedades. ${ }^{1}$ La epidemia de esta

\footnotetext{
* Docente-Investigador de la Unidad Académica de Odontología de la Universidad Autónoma de Zacatecas «Francisco García Salinas».
}

Recibido: 09 Abril 2020.

Aceptado para publicación: 20 Abril 2020.

\section{ABSTRACT}

The emergence of human infections caused by SRAS-CoV-2 has triggered a series of health, economic and social conflicts in the global environment. The dental area must have all the necessary knowledge about this pandemic because, as a health professional and responsible for the oral condition of society, it is vitally important to reduce the risk of chronic non-communicable diseases, cancer, obesity and others, as well as older adults so that they do not generate a health problem and that may also be associated with the presence of COVID-19. It is important to know the problems of the guidelines to consider in dental practice for effective dental care and biosafety of health personnel. Therefore, this dental care guide is generated based on published scientific evidence for the knowledge and domain of the oral health professional.

Keywords: COVID-19, coronavirus, dentistry, management, biosafety, public health. enfermedad (COVID-19) comenzó en Wuhan, Provincia de Hubei, de la República Popular China en diciembre de 2019 como un brote de neumonía de causa desconocida. Esta situación se informó ante la Organización Mundial de la Salud (OMS) mediante la presencia de 27 casos de síndrome respiratorio agudo (SRA) de etiología desconocida, considerándose como posible vínculo el mercado mayorista de mariscos de Huanan, el cual en su mayoría se dedica a la venta de animales vivos. ${ }^{2}$ Esta enfermedad se ha convertido en uno de los mayores retos de salud pública, no sólo para China sino a nivel mundial. ${ }^{3}$

Esta circunstancia generó que científicos chinos aislaran y caracterizaran mediante secuenciación genética esta 
nueva cepa de coronavirus, que corresponde a un virus que pertenece a los betacoronavirus del grupo $2 \mathrm{~B}$ con al menos un $70 \%$ de similitud en la secuencia genética con el virus del síndrome respiratorio agudo severo (SARS-CoV), el cual fue nombrado como 2019-nCoV por la OMS. ${ }^{4}$

El nuevo coronavirus se une a los receptores de las células diana a través de una proteína $S$ facilitando la entrada en las mismas. Se ha identificado a la proteína receptora celular de la enzima convertidora de angiotensina 2 (ACE2) relacionada con la esterasa de la hemaglutinina (HE). En un estudio más reciente, Wang y colaboradores demostraron que la molécula CD147 sirve como receptor del virus en las células huésped como un mediador de la infección viral. ${ }^{5}$ Se han identificado dos cepas de COVID-19, la L más agresiva (70\% de casos en China) y la S (menos agresiva con un $30 \%$ de casos). Se ha reportado que el virus puede permanecer viable en aerosoles durante tres horas, en cobre durante cuatro horas y en plásticos y acero inoxidable hasta por 72 horas. ${ }^{6}$

El 30 de enero de 2020 la OMS consideró que este brote se estimaba como un problema de salud pública de emergencia internacional, el cual fue renombrado como SARS-CoV-2 por su similitud con el virus del SARS. El 28 de marzo el COVID-19 era detectado en 177 países con un total de 571,678 casos confirmados por laboratorio y 26,494 muertes según el reporte de la OMS. ${ }^{7}$ El primer caso de coronavirus en México se detectó el 28 de febrero como un caso importado en un hombre de 35 años de la Ciudad de México, que contaba con antecedente de viaje a Italia, el segundo país con más infectados después de China en ese momento. ${ }^{8}$ En México, hasta el momento de finalizar esta publicación, se presentaban un total de 3,181 casos confirmados y 174 defunciones por COVID-19. ${ }^{9}$ Estos datos irán en aumento o descenso de acuerdo con las características de propagación del virus a nivel mundial.

Los casos confirmados de COVID-19 hasta el día de hoy sugieren que la evolución de la enfermedad no es igual a la reportada por el SARS-CoV y MERS-CoV. Sin embargo, se ha observado que los adultos mayores, especialmente con comorbilidades como enfermedad coronaria, hipertensión, diabetes mellitus, personas inmunocomprometidas y mujeres embarazadas pueden tener un mayor riesgo de presentar enfermedad grave por este virus. ${ }^{10}$

Los síntomas principales son: fiebre mayor a $38{ }^{\circ} \mathrm{C}$ (> 90\% de los casos), tos seca $(80 \%)$, dolor torácico (20\%), dificultad respiratoria (15\%) y malestar general. ${ }^{11}$ $\mathrm{Si}$ el paciente cumple con los dos primeros síntomas y ha estado en contacto con una persona confirmada de
COVID-19 se realiza una prueba molecular confirmatoria mediante la toma de muestra faríngea y nasofaríngea con un hisopo y se analiza por reacción en cadena de la polimerasa con transcriptasa reversa (RT-PCR, por sus siglas en inglés) para confirmar o descartar la presencia del virus. En una tomografía computarizada (TC) de tórax se observa opacidad parecida al «vidrio esmerilado», sombreado irregular local, sombreado irregular bilateral o anomalías intersticiales. ${ }^{12}$

Debido a las características del entorno odontológico, el riesgo de infección cruzada puede ser bastante alto entre odontólogos y pacientes. En países o regiones donde la práctica odontológica y hospitalaria se encuentra o será afectada por el COVID-19, es necesaria la implementación de protocolos inmediatos y efectivos. ${ }^{13}$ La Norma Oficial Mexicana NOM-013-SSA2-2015 para la prevención y el control de las enfermedades bucales contempla en el apartado 5.19 que el estomatólogo debe considerar a todo paciente y personal potencialmente infeccioso, y en el apartado 5.10 que el personal de estomatología debe dominar y practicar los procedimientos de control de infecciones y seguridad del paciente en el establecimiento de atención odontológica. ${ }^{14}$ Sin embargo, las cifras seguirán aumentando a pesar de las estrategias que se han implementado para contener la propagación del coronavirus en nuestro país. Se ha observado que la evolución de la enfermedad a nivel global tiene un crecimiento exponencial, por lo cual es necesario proporcionar herramientas útiles para la prevención y control del cuadro viral en la clínica odontológica. La presente guía ha sido elaborada con base en la información actualizada disponible en las diferentes bases de datos como PubMed, Scopus, Google Académico y diferentes páginas oficiales del gobierno mexicano, lo cual ayudará al estudiante, odontólogo de práctica general y especialista a tener una guía para el conocimiento y prevención de la enfermedad mediante las diferentes estrategias que se recomiendan en la consulta odontológica ante la presencia del COVID-19.

\section{EPIDEMIOLOGÍA}

De acuerdo con los hallazgos genéticos y epidemiológicos reportados por la literatura, parece que el COVID-19 comenzó como una transmisión de animal a humano mediante la ingesta del murciélago chino (Rhinolophus sinicus) y después de humano a humano. ${ }^{15}$ En la actualidad, se ha demostrado que la transmisión ocurre mediante las gotas de saliva de un infectado con la mucosa (nasal, oral y ocular) de otro sujeto y por contacto con heces feca- 
les. ${ }^{16}$ Otra vía de transmisión es mediante la dispersión de aerosoles contaminados o de madre a recién nacido (transmisión vertical). ${ }^{17}$

En cuanto a la transmisión, a pesar de que los pacientes sintomáticos se han considerado como los principales vectores de transmisión, observaciones recientes sugieren que pacientes asintomáticos y pacientes en periodo de incubación también son fuentes de transmisión del SARS$\mathrm{CoV}-2 .{ }^{18}$ Se ha reportado un periodo de incubación de cinco a seis días en promedio, aunque hay evidencia de que puede durar hasta 14 días, de ahí la importancia de aislar a los sujetos sospechosos de contagio durante esos días. ${ }^{19}$ La tasa de mortalidad se ha estimado a nivel mundial entre un 0.39 a $4.05 \%$, en comparación con el virus del SARS con un promedio del $10 \%$, con el MERS de $34 \%$ y con el virus de la influenza con un 0.01 a $0.17 \%$ de acuerdo con los datos de 2010 a 2017 del Centro para el Control y Prevención de Enfermedades (CDC) de los Estados Unidos. ${ }^{11}$ En este contexto es necesaria una evidencia más robusta para definir grupos con riesgo para presentar complicaciones graves asociadas a esta enfermedad. Los grupos de alto riesgo ${ }^{20}$ a contraer la infección son:

a) Niños menores de 5 años, especialmente menores de 2 años.

b) Adultos $\geq 65$ años.

c) Enfermedad pulmonar obstructiva crónica (EPOC), fibrosis quística e incluso asma.

d) Enfermedad cardiovascular (excepto hipertensión arterial aislada).

e) Nefropatía.

f) Hepatopatía.

g) Enfermedad hematológica (incluyendo anemia falciforme).

h) Alteraciones metabólicas (incluyendo diabetes mellitus tipo 1, 2 y gestacional; incluso bajo control).

i) Afección neurológica (incluyendo afección cerebral, espinal, nervios periféricos, parálisis, síndromes epilépticos, evento vascular cerebral y afección intelectual) o del neurodesarrollo.

j) Inmunosupresión inducida por medicamentos.

k) Personas con VIH positivo.

l) Personas que viven en asilos $u$ otros centros de cuidados a largo plazo.

m) Mujeres embarazadas y hasta dos semanas del postparto.

n) Personas que tienen obesidad, con un índice de masa corporal $^{21} \geq 40$.

o) Personal de salud.

\section{MANIFESTACIONES CLÍNICAS}

La mayoría de las manifestaciones son leves-moderadas. En China se ha observado una proporción para casos severos entre todos los pacientes infectados de 15$25 \% .^{22}$ Los síntomas se han clasificado de la siguiente manera de acuerdo con la gravedad de la enfermedad (Tabla 1). ${ }^{21}$

\section{Diagnóstico}

El criterio operacional de diagnóstico de acuerdo con los lineamientos para la atención de pacientes por COVID-19 de la Secretaría de Salud en México ${ }^{8}$ se observa en la Figura 1.

\section{Tratamiento}

El abordaje para el manejo de la enfermedad, de acuerdo con los lineamientos para la atención de pacientes por COVID-19 de la Secretaría de Salud en México, se observa en la Figura 2.

\section{PREVENCIÓN Y CONTROL EN LA CONSULTA ODONTOLÓGICA}

De acuerdo con Peng y colaboradores ${ }^{23}$ el riesgo y ruta de transmisión de infección en el consultorio puede estar latente por pacientes con las siguientes características:

Tabla 1: Sintomatología de pacientes sospechosos de COVID-19 dependiendo del grado de enfermedad.

\begin{tabular}{|c|c|}
\hline Leve-moderado & Grave \\
\hline Fiebre $\geq 38^{\circ} \mathrm{C}$ & Disnea \\
\hline Tos seca & $\begin{array}{l}\text { Oximetría de pulso }<94 \% \text { al aire } \\
\text { ambiente }\end{array}$ \\
\hline Artralgia & Abundantes secreciones \\
\hline Mialgia & Taquipnea \\
\hline $\begin{array}{l}\text { Odinofagia/ardor } \\
\text { faríngeo }\end{array}$ & Síndrome pleuropulmonar \\
\hline Rinorrea & Hipotensión arterial \\
\hline Conjuntivitis & $\begin{array}{l}\text { Exacerbaciones de síntomas cardio- } \\
\text { vasculares o respiratorios de enfer- } \\
\text { medades crónicas subyacentes }\end{array}$ \\
\hline Dolor torácico & $\begin{array}{l}\text { Trastornos del estado de conciencia } \\
\text { Vómito o diarrea persistente } \\
\text { Descontrol glucémico }\end{array}$ \\
\hline
\end{tabular}




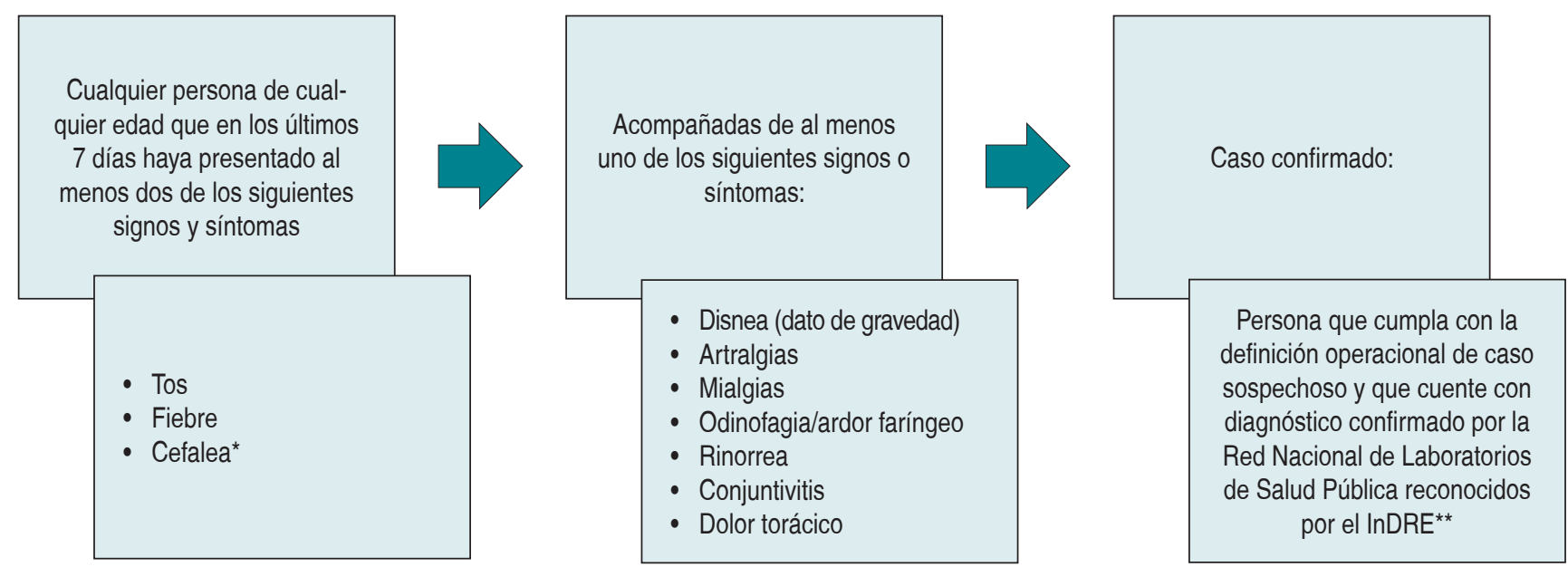

Figura 1: Criterio operacional para el diagnóstico clínico. * En niños la irritabilidad sustituye a la cefalea. **InDRE = Instituto de Diagnóstico y Referencia Epidemiológica.

tos, estornudos, tratamientos con turbina de alta velocidad, uso de instrumentos ultrasónicos, antecedentes de viaje y/o contacto con alguna persona que lo haya realizado, contacto con personas con familiares en las situaciones antes descritas. Además de ser propensos a dispersar secreciones se sangre o saliva los pacientes con aparatología fija o removible, así como pacientes que no se hayan realizado el lavado de manos previo a la consulta.

Se debe considerar que de acuerdo a la información del Comité Nacional para la Vigilancia Epidemiológica (CONAVE), esta pandemia de COVID-19, muy probablemente tenga su permanencia en territorio mexicano hasta el mes de agosto o septiembre, lo que nos lleva al escenario real de que las urgencias y padecimientos dentales actualmente están en la población y afectando a los pacientes de alta vulnerabilidad (HTA, diabetes mellitus, pacientes inmunológicamente deprimidos, obesidad, embarazo, etcétera), por lo que el odontólogo y especialista no pueden parar ni omitir la atención de pacientes por ese periodo tan largo de tiempo; finalmente, si no hay atención de un procedimiento o urgencia dental, el estado sistémico de estos pacientes se puede deteriorar de importante manera y no precisamente por la pandemia del COVID-19. Es importante recalcar que se describe un protocolo basado en la evidencia publicada de cómo realizar un procedimiento efectivo para controlar el riesgo de infección no sólo por COVID-19, sino además por la gran cantidad de riesgos que cotidianamente existen en un consultorio odontológico (Tabla 2). ${ }^{24}$

\section{MEDIDAS RECOMENDABLES DURANTE EL BROTE DE COVID-19}

\section{Recomendaciones del manejo}

En enero de 2020, la Comisión Nacional de Salud en China clasificó al COVID-19 en la categoría de enfermedades infecciosas del grupo B, que incluye al SARS e influenza aviaria. Se sugiere que se maneje de una manera similar a aquellas infecciones clasificadas en el grupo A, una categoría reservada para patógenos infecciosos extremos como cólera y la plaga. ${ }^{25}$

\section{Recomendaciones para la atención odontológica}

Prioritario: evaluar sintomatología de todo el personal involucrado y toma de temperatura $<37^{\circ} \mathrm{C}$

Debido a la situación epidemiológica actual en nuestro país, nos vemos con la responsabilidad y obligación de realizar una correcta anamnesis a nuestros pacientes. Las siguientes preguntas servirán para que el profesional tome una decisión adecuada y, de esta manera, proceder o no con los distintos tratamientos de urgencia mediante los siguientes puntos generales:

a) Estado general de salud y viajes o contacto con alguien que haya viajado al extranjero en los últimos 14 días, aplica también para acompañantes de los pacientes.

b) Temperatura $<37^{\circ} \mathrm{C}$.

c) En áreas donde se haya expandido el virus, cualquier procedimiento de rutina deberá posponerse. 
d) Pacientes que hayan presentado la infección por COVID-19, se sugiere posponer los procedimientos hasta un mes después de aliviados los síntomas.

\section{Cuestionario}

a) En este momento zusted presenta fiebre, falta de aire, dolor de cabeza o la ha tenido en los últimos 14 días?

b) ¿Ha tenido algún problema respiratorio (incluyendo tos seca) en los últimos 14 días?

c) ¿Ha estado en contacto estrecho con personas que presenten o presentaban algún cuadro respiratorio en los últimos 14 días?

d) ¿Usted o alguien en contacto directo con usted ha estado con alguna persona con confirmación del coronavirus?

e) ¿Usted o alguien en contacto directo con usted viajó a países de riesgo durante el brote del coronavirus?

\section{Toma de decisiones}

Considerando el cuestionario anterior, el odontólogo deberá tomar la mejor opción tomando en cuenta lo descrito en la Figura 3.

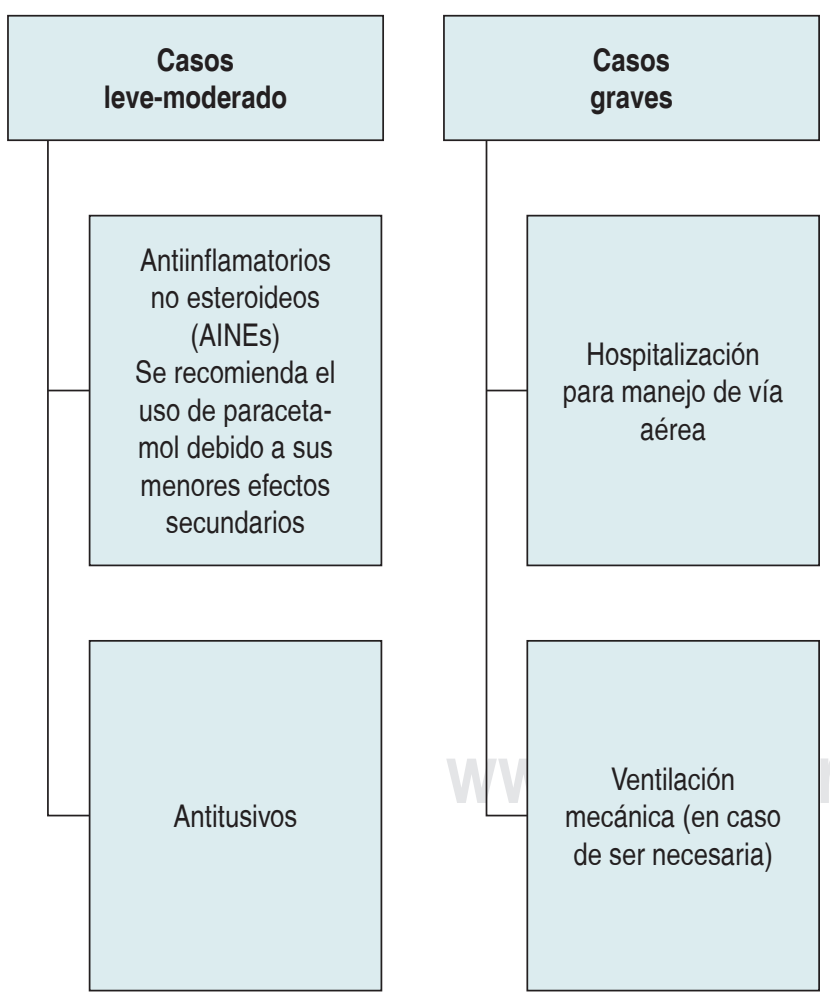

Figura 2: Tratamiento para la sintomatología del COVID-19.

\section{Tabla 2: Procedimientos efectivos para el control de la} infección en consultorio de práctica dental.

A) Lavado de manos:

1. Agua y jabón (30 s)

2. Secado con toallas desechables

3. Reforzar lavado de manos antes y después de cada paciente

4. Aplicar gel a base hidroalcohólica $(\geq 70 \%)$

B) Limpieza de superficies:

1. Con alcohol $(\geq 70 \%)$

2. Con $\mathrm{NaOCl}\left(10 \mathrm{~mL}\right.$ de solución $/ 1,000 \mathrm{~mL}$ de $\left.\mathrm{H}_{2} \mathrm{O}\right)$

3. Retirar de sala de espera libros, revistas y material de lectura

C) Barreras de protección personal:

1. Cubrebocas (preferentemente N95)

2. Guantes

3. Bata desechable

4. Gorro desechable

5. Careta de protección

6. Manejo de protocolo de RPBI

7. Uso constante de eyectores de saliva y/o quirúrgicos (preferentemente)

D) Para la práctica dental:

Se debe recordar que la recomendación de la OMS es sólo dar atención a URGENCIAS DENTALES, pero algunos procedimientos requieren el uso de turbina de alta velocidad, en estos casos se recomienda lo siguiente:

1. Técnica de 4 manos (eficacia y menor tiempo de atención)

2. Enjuague bucal a base de alcohol $(60 \%)$

3. Clorar el agua del recipiente de la unidad dental $(5 \mathrm{ml}$ de solución $/ 500 \mathrm{~mL}$ de $\mathrm{H}_{2} \mathrm{O}$ destilada)

4. Eficacia en la técnica anestésica y toma radiográfica

\section{Evaluación bucal}

a) Enjuagues bucales preoperatorios (con contenido alcohólico o antisépticos orales) pueden reducir el número de microorganismos. Evitar enjuagues con hipoclorito de sodio (tóxico e irritante).

b) Procedimientos que sean afines a provocar tos deberán evitarse (si es posible) o realizarlos cuidadosamente.

c) Minimizar el uso de la jeringa triple en la medida de lo posible.

d) Las radiografías intraorales pueden estimular la secreción de saliva, tos o reflejo nauseoso. 


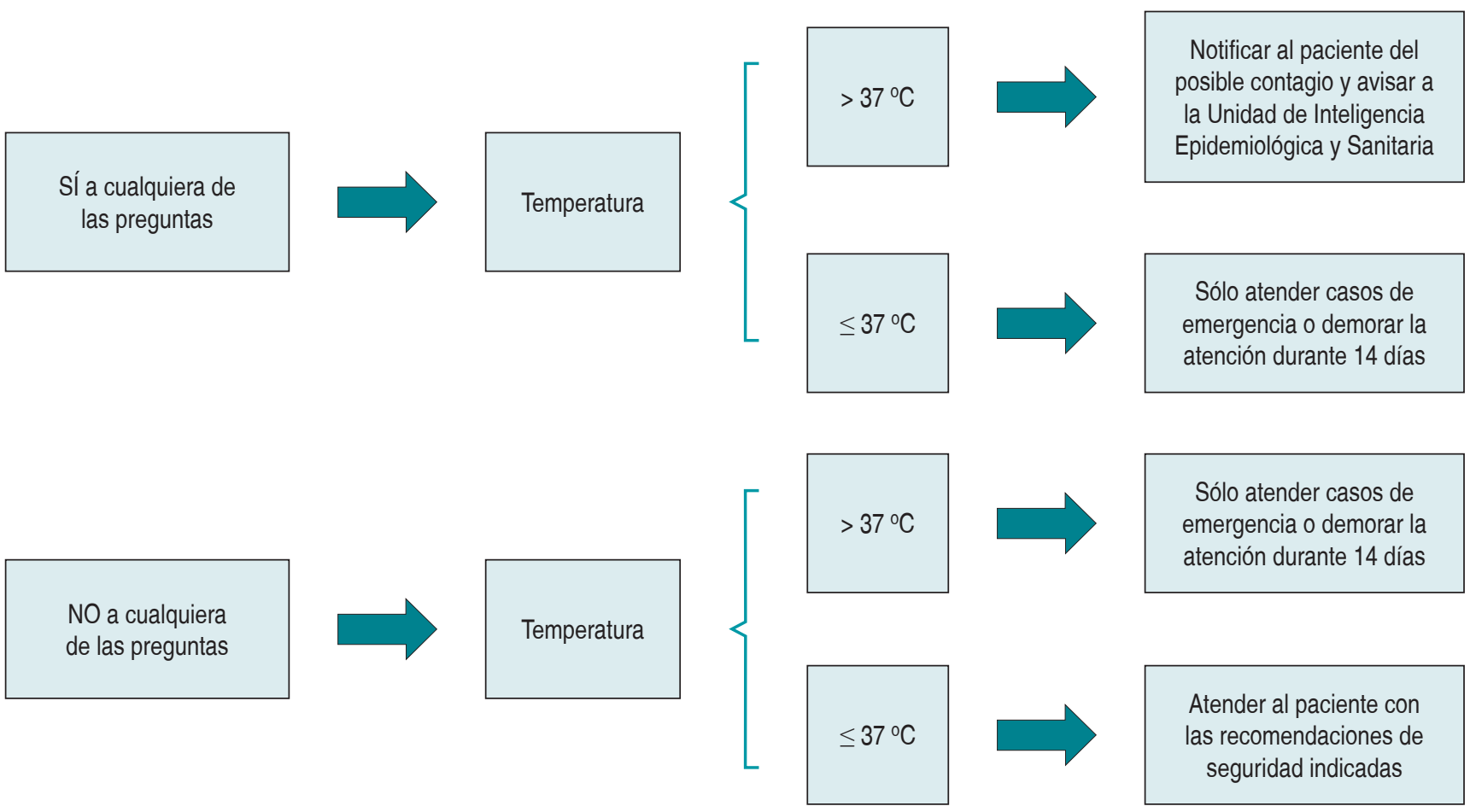

Figura 3: Esquema para toma de decisión en el tratamiento dental. Tomado y modificado de: Consejo General de Dentistas de España 2020. ${ }^{26}$

e) Se recomienda el uso de radiografías panorámicas o tomografía Cone Beam si es posible durante el brote del virus.

\section{Tratamiento de casos emergentes}

Los siguientes escenarios clínicos se clasifican como urgencias odontológicas:

a) Pulpitis reversible e irreversible.

b) Absceso periapical agudo.

c) Absceso periodontal.

d) Periodontitis apical aguda.

e) Celulitis.

f) Trauma dental (excepto aquéllos que involucren esmalte o dentina sin la presencia de dolor).

g) Pericoronitis.

h) Sangrado oral.

i) Sospecha de lesión patológica maligna.

j) Fractura o pérdida de restauraciones asociada con dolor.

k) Fractura o pérdida de prótesis fija o removible con la presencia de dolor o infección.

I) Retiro de sutura asociada con procedimiento oral reciente. m) Dolor espontáneo o limitación de la apertura (trauma o trismus).

n) Luxación o fractura de la articulación temporomandibular.

\section{Protocolos de seguridad}

a) Utilizar siempre aislamiento absoluto con dique de hule (sellar todas las filtraciones) y eyector de saliva (de preferencia quirúrgico) para evitar o minimizar la dispersión de saliva.

b) Lentes de seguridad y/o mascarilla de protección (preferente) para el operador y asistente, sobre todo si se va a utilizar pieza de alta velocidad, ultrasonido o jeringa triple.

c) En caso de pulpitis irreversible se recomienda el uso de agentes quimio-mecánicos para el acceso y drenar. Posponer en la medida de lo posible la obturación final hasta que el brote haya bajado.

d) En fracturas, luxaciones o avulsiones dentales evaluar la severidad traumática del tejido, el desarrollo del ápice y la duración del tiempo de avulsión en cualquiera de los casos.

e) Minimizar en la medida de lo posible el procedimiento quirúrgico. Evitar o minimizar la dispersión de saliva, 
sangre o agua y en caso de sutura se recomienda que sea absorbible.

\section{CONCLUSIONES Y RECOMENDACIONES PARA LA SALUD ORAL}

- Es prioritaria la comunicación entre personal de práctica privada, clínicas universitarias y hospitales con el Departamento de Epidemiología local, estatal o nacional, así como de los estudiantes con sus profesores clínicos y epidemiólogos.

- El personal administrativo deberá estar siempre informado y capacitado para mejorar y facilitar la confianza, así como una cooperación de todos los niveles.

- Durante el brote se recomienda que los estudiantes y profesores clínicos realicen investigación bibliográfica actual, estudios de casos, conferencias informativas de divulgación y científicas, atención a noticieros nacionales y sobre todo en cuanto a los comunicados de las autoridades de salud en todo momento.

- Como profesionales de la salud, estamos obligados a educar a los pacientes, familiares y conocidos en cuanto a las medidas de prevención, de diagnóstico y tratamiento para eliminar mitos sociales y pánico generalizado.

- Es importante recordar que, al ser formados académica y clínicamente, tenemos todos los conocimientos necesarios para el manejo de la bioseguridad en el consultorio odontológico, por lo que ante esta pandemia la seguridad y confianza debe predominar en nuestra persona.

\section{BIBLIOGRAFÍA}

1. Li Q, Guan X, Wu P, Wang X, Zhou L, Tong Y et al. Early transmission dynamics in Wuhan, China, of novel coronavirus-infected pneumonia. N Engl J Med. 2020; 382 (13): 1199-1207.

2. Risk assessment-pneumonia Wuhan, China: 2020 [26 march 2020]. Available from: http://ecdc.europa.eu/sites/default/files/documents/ Risk\%20assessment\%20\%20pneumonia\%20Wuhan\%20China\%20 17\%20Jan\%202020.pdf.

3. Phelan AL, Katz R, Gostin LO. The novel coronavirus originating in Wuhan, China: challenges for global health governance. JAMA. 2020 Jan 30. doi: 10.1001/jama.2020.1097.

4. Hui DS, I Azhar E, Madani TA, Ntoumi F, Kock R, Dar O et al. The continuing 2019-nCoV epidemic threat of novel coronaviruses to global health - The latest 2019 novel coronavirus outbreak in Wuhan, China. Int J Infect Dis. 2020; 91: 264-266.

5. Wang K, Chen W, Zhou YS, Lian JQ, Zhang Z, Du P et al. SARSCoV-2 invades host cells via a novel route: CD147-spike protein. bioRxiv. 2020.
6. van Doremalen N, Bushmaker T, Morris DH, Holbrook MG, Gamble A, Williamson BN et al. Aerosol and surface stability of SARS-CoV-2 as compared with SARS-CoV-1. N Engl J Med. 2020; 382 (16): 1564-1567.

7. World Health Organization. Coronavirus disease 2019 (COVID-19). Situation report-68. Available from: https:/www.who.int/docs/ default-source/coronaviruse/situation-reports/20200328-sitrep-68covid-19.pdf?sfvrsn=384bc74c_8

8. Aviso Epidemiológico. Enfermedad COVID-19 por SARS-CoV-2. 17 de marzo 2020 [acceso 17 marzo 2020]. Disponible en: https://www.gob.mx/cms/uploads/attachment/file/541794/ AE_Enfermedad_COVID-19_SARS-CoV-2_2020.03.17.pdf.

9. Secretaría de Prevención y Promoción de Ta Salud. Comunicado técnico diario nuevo coronavirus en el mundo (COVID-19). 2020 [08 abril 2020]. Disponible en: https://www.gob.mx/cms/ uploads/attachment/file/545943/Comunicado_Tecnico_Diario_ COVID-19_2020.04.08.pdf.

10. Novel Coronavirus in Hubei Province, China [26 march 2020]. Available from: https://wwwnc.cdc.gov/travel/notices/warning/ novel-coronavirus-wuhan-china.

11. Malik YS, Sircar S, Bhat S, Sharun K, Dhama K, Dadar M et al. Emerging novel Coronavirus (2019-nCoV)-Current scenario, evolutionary perspective based on genome analysis and recent developments. Vet Q. 2020; 40 (1): 68-76.

12. Ai T, Yang Z, Hou H, Zhan C, Chen C, Lv W et al. Correlation of chest CT and RT-PCR testing in coronavirus disease 2019 (COVID-19) in China: a report of 1014 cases. Radiology. 2020; 200642.

13. Meng L, Hua F, Bian Z. Coronavirus disease 2019 (COVID-19): emerging and future challenges for dental and oral medicine. J Dent Res. 2020; 99 (5): 481-487.

14. NORMA Oficial Mexicana NOM-013-SSA2-2015, Para la prevención y control de enfermedades bucales. Diario Oficial de la Federación 2016. Disponible en: https://dof.gob.mx/nota_detalle. php? codigo $=5462039 \&$ fecha $=23 / 11 / 2016$.

15. Chan JFW, Yuan S, Kok KH, To KKW, Chu H, Yang J et al. A familial cluster of pneumonia associated with the 2019 novel coronavirus indicating person-to-person transmission: a study of a family cluster. Lancet. 2020; 395 (10223): 514-523.

16. Rothan HA, Byrareddy SN. The epidemiology and pathogenesis of coronavirus disease (COVID-19) outbreak. J Autoimmun. 2020; 109: 102433.

17. Zhu H, Wang L, Fang C, Peng S, Zhang L, Chang G, et al. Clinical analysis of 10 neonates born to mothers with 2019-nCoV pneumonia. Transl Pediatr. 2020; 9 (1): 51-60.

18. Bai Y, Yao L, Wei T, Tian F, Jin DY, Chen L et al. Presumed asymptomatic carrier transmission of COVID-19. JAMA. 2020. doi: 10.1001/jama.2020.2565.

19. Berry A, Fillaux J, Martin-Blondel G, Boissier J, Iriart X, Marchou $B$ et al. Evidence for a permanent presence of schistosomiasis in Corsica, France, 2015. Euro Surveill. 2016; 21 (1).

20. Grohskopf LA, Sokolow LZ, Broder KR, Walter EB, Fry AM, Jernigan DB. Prevention and control of seasonal influenza with vaccines: recommendations of the advisory committee on immunization practices-united states, 2018-19 influenza season. MMWR Recomm Rep. 2018; 67 (3): 1-20.

21. Secretaría de Salud, Gobierno de México. Lineamiento para la atención de pacientes por COVID-19. 14 febrero 2020. Disponible en: https://drive.google.com/file/d/1vge89Fuz_9RsgKk77XrpyG2R YW7NAGFP/view.

22. Guan WJ, Ni ZY, Hu Y, Liang WH, Ou CQ, He JX et al. Clinical characteristics of 2019 novel coronavirus infection in China. MedRxiv. 2020. 
23. Peng X, Xu X, Li Y, Cheng L, Zhou X, Ren B. Transmission routes of 2019-nCoV and controls in dental practice. Int J Oral Sci. 2020; 12 (1): 9.

24. Kohn WG, Collins AS, Cleveland JL, Harte JA, Eklund KJ, Malvitz DM; Centers for Disease Control and Prevention (CDC). Guidelines for infection control in dental health-care settings--2003. MMWR Recomm Rep. 2003; 52 (RR-17): 1-61.

25. Li ZY, Meng LY. The prevention and control of a new coronavirus infection in department of stomatology. Zhonghua Kou Qiang Yi Xue Za Zhi. 2020; 55 (0): E001.

26. Organización Colegial de Dentistas de España. El nuevo Coronavirus 2019-nCOV y el manejo del paciente dental. Marzo 2020. Disponible en: https://gacetadental.com/wp-content/
uploads/2020/03/INFORME-TE\%CC\%81CNICO-DEL-CONSEJOGENERAL.pdf.

\section{Correspondencia:}

\section{Carlos Omar Bermúdez Jiménez}

Juan G. Estrada 116-B,

Col. Médicos Veterinarios, 98097,

Zacatecas, Zacatecas, México.

Teléfono: 4929463419

E-mail: carlosbj8@gmail.com 